\title{
Entretien avec Chantal Desbordes, Contre-Amiral de la Marine française
}

\author{
Luc Capdevila et Dominique Godineau
}

\section{(2) OpenEdition \\ 1 Journals}

Édition électronique

URL : https://journals.openedition.org/clio/1391

DOI : 10.4000/clio. 1391

ISSN : 1777-5299

Éditeur

Belin

Édition imprimée

Date de publication : 1 novembre 2004

Pagination : 193-210

ISBN : 2-85816-755-9

ISSN : 1252-7017

Référence électronique

Luc Capdevila et Dominique Godineau, «Entretien avec Chantal Desbordes, Contre-Amiral de la Marine française ", Clio. Histoire, femmes et sociétés [En ligne], 20 | 2004, mis en ligne le 01 janvier 2007, consulté le 23 avril 2022. URL : http://journals.openedition.org/clio/1391 ; DOI : https://doi.org/ 10.4000/clio.1391

Ce document a été généré automatiquement le 22 avril 2022.

Tous droits réservés 


\title{
Entretien avec Chantal Desbordes, Contre-Amiral de la Marine française
}

\author{
Luc Capdevila et Dominique Godineau
}

Clio: Pourriez vous nous préciser les circonstances dans lesquelles vous avez été amenée à vous orienter vers une carrière militaire?

$\mathrm{CD}:$ Au risque de décevoir ceux qui m'imaginent animée d'une motivation militaire et maritime exemplaire, je dois avouer que je n'avais pas du tout envisagé cette opportunité de carrière. Je voulais faire du cinéma, mais c'était - et cela reste - un milieu difficile à pénétrer. J'ai donc entrepris des études supérieures, en espérant que la conjoncture s'améliore. C'est alors que j'ai eu connaissance dans la presse locale d'un recrutement sur titres d'officiers féminins. J'ai constitué un dossier et, à ma grande surprise, la Marine m'a fait savoir que ma candidature l'intéressait. C'était en 1969, après l'obtention d'une licence d'enseignement de lettres classiques à la faculté de Tours. Je me suis rendue à Paris. Là, nous étions huit jeunes femmes en course - à l'origine, nous devions être une quarantaine pour l'ensemble de la France - : on nous a examinées sous toutes les coutures, et à la fin de la sélection, deux d'entre nous ont été retenues. Si je me suis engagée dans cette voie, c'est parce que le contrat n'était que de trois ans. À l'époque, les officiers féminins n'étaient pas encore militaires de carrière. Alors, trois ans me permettaient d'entrer dans la vie active.

Clio : En quelque sorte, vous êtes entrée dans l'armée pour ne pas vous engager?

CD : On pourrait presque l'énoncer ainsi, comme un paradoxe! En fait, ce qui m'a décidée à signer, c'est de savoir qu'à l'issue de la formation initiale, la première de nous deux pourrait choisir son affectation et que la Marine venait d'ouvrir un poste dans son service d'information et de relations publiques (SIRPA). Poursuivant mon idée de métier, je me suis dit qu'il fallait que je travaille pour être la première et décrocher ce poste-là. En effet, si j'intégrais le SIRPA, j'aurais un pied dans la place pour approcher le journalisme, la télévision et, qui sait ? le cinéma. Donc, trois ans donneraient peut-être au cinéma le temps de sortir de son éternelle crise et à moi, le moyen de l'aborder plus 
favorablement. Je dois ajouter que servir mon pays sous l'uniforme était loin de me déplaire.

Clio: Avez-vous eu le sentiment, à ce moment-là, de choisir un métier d'homme, voire d'être une pionnière?

CD : Pas d'être une pionnière. Absolument pas. Je n'avais pas du tout prémédité cette carrière. Mais de faire un métier d'homme ? Oui. D'abord dans l'appellation : officier de la Marine. Puis, et je m'en rends compte dès mon arrivée à Brest, le $1^{\mathrm{er}}$ novembre 1970, par un jour de grisaille, le sentiment d'être projetée dans un environnement où l'on ne voit pas de femmes. Quantitativement, nous sommes alors en tout et pour tout 196 femmes, dont 10 officiers, pour 75000 hommes, dont 6000 officiers. Inutile de dire que nous n'« existons » pas, nous sommes un groupe microscopique! Pourquoi la Marine recrute-t-elle des officiers féminins? Essentiellement pour recruter, former, administrer, gérer le personnel féminin non officier, dans des structures spécifiquement féminines, en quelque sorte placées en marge de l'institution. Bien sûr, ces jeunes officiers mariniers (sous-officiers) tiennent parfaitement leurs emplois, mais ce sont des emplois subalternes et «traditionnels" d'assistantes, de secrétaires (d'autant plus appréciées qu'elles ont un joli sourire) et nous, officiers féminins, sommes là pour les encadrer dans une sorte de direction des ressources humaines... féminine !

7 Clio: Comment avez-vous vécu cette situation d'isolement? vous retrouver seule femme dans un milieu d'hommes, au sein d'une institution, l'armée, dont l'image est associée au masculin?

$\mathrm{CD}$ : Je crois très sincèrement que j'avais en moi une combativité bien utile. Elle a sans doute été suscitée par l'éducation familiale, et puis je l'ai développée peut-être au-delà de la norme grâce au parcours que j'ai fait. Mais il y a autre chose, quelque chose de plus subtil, à quoi je dois faire référence ici : c'est la part de masculin et de féminin que chacun porte en soi. Je pense qu'inconsciemment, j'avais le désir de côtoyer, et même d'affronter du masculin et que ce monde d'hommes allait aussi me reconduire à ma part de féminité. Nous avons besoin du miroir des autres pour nous connaitre. Il est important de voir comment fonctionne l'autre, pour le comprendre et pour se comprendre soi-même. Cette complémentarité est indispensable. Si les femmes sont généralement analytiques, sérieuses et précises (l'esprit de finesse), les hommes avec leur esprit de géométrie ont bien des qualités aussi. J'ai beaucoup appris à leur contact, j'aime vraiment travailler avec eux ; j'apprécie en particulier leur sens de la synthèse, parfois un peu approximatif, leur capacité à conceptualiser, tout cela m'a considérablement apporté.

Clio : Dans les années 1970, quel était le quotidien des femmes dans l'armée, vous étiez regroupées entre vous?

CD : Oui, je dirais : cantonnées dans certains emplois. Pour ma part, je n'aurai pas la même carrière que les neuf officiers qui me précèdent et qui toutes, excepté deux affectées comme secrétaires particulières d'amiraux, se consacrent à la gestion du personnel. Pour mon premier poste, ici, rue Royale, au SIRPA/ Marine, je m'emploie à faire connaître la Marine à l'extérieur. On sait que je suis littéraire de formation, donc on utilise ces compétences-là. Je dois écrire sur les missions et les moyens de la Marine, rédiger des dépliants, des plaquettes, des brochures destinés au grand public. Cela présuppose que je connaisse un peu mon sujet. Le connaître, ça veut dire aller voir la Marine au plus près. Je suis donc reçue à bord de tous les types de bateaux. J'accompagne également des parlementaires, des enseignants, des visiteurs de tous les 
horizons socioprofessionnels. Y compris à bord des sous-marins stratégiques. C'est une plus-value par rapport à mes anciennes, car j'acquiers ainsi une connaissance de la Marine très large, quoique superficielle. En effet, les femmes ne sont pas admises sur les bâtiments : dès qu'il faut appareiller, on me reconduit à quai immédiatement. Je ne suis tolérée à bord que le temps d'un thé ou d'un déjeuner, quand j'accompagne des hôtes ou lorsque j'enquête pour ensuite écrire en connaissance de cause. Mais ce faisant, je ne touche pas le cœur du métier: c'est une vision à la fois directe et distanciée.

11 Clio: Vous avez évoqué des résistances très fortes au sein de l'armée. Vous disiez au début que vous vous sentiez illégitime. Cela ne vous a pas empêché de beaucoup travailler, d'obtenir la reconnaissance, et de réussir une belle carrière.

12 CD : Je me suis tout de suite infiniment plu dans le milieu de la Marine, que j'ai trouvé original par rapport à ce que je pouvais connaître du monde et de la vie. J'ai aussi perçu sa dimension majoritairement masculine. D'ailleurs, ce point mérite un court développement. Quand on est femme et que l'on appartient au groupe minoritaire, il faut apprendre à gérer son propre comportement par rapport aux hommes, les plus nombreux. J'ai vécu cela non pas comme de la soumission, mais comme une nécessaire adaptation : dans ce rapport de force ou de nombre - on pourrait faire un parallèle avec les problématiques de l'exclusion et de l'intolérance - je regrette de constater que c'est à la minorité de s'adapter à la majorité, et rarement l'inverse. Il m'a fallu intégrer cette réalité-là très vite et, par exemple, ne pas tomber dans le piège du militantisme féministe alors très présent dans la société civile, ou bien me protéger du désir de familiarité de certains, ou de l'attitude misogyne de certains autres.

13 Mes débuts avec mes supérieurs ont souvent été rudes. J'analyse ce fait comme une donnée " générationnelle » (des hommes n'ayant pas vécu la mixité à l'école) à laquelle s'ajoute l'exercice d'un métier «entre hommes ». Si quelques-uns avaient connu des secrétariats féminins, aucun n'avait travaillé avec des femmes cadres, des officiers subalternes puis supérieurs. Dans ces conditions, on peut comprendre leur appréhension, voire leur malaise, d'où des relations tendues au départ. Je me souviens de l'un d'entre eux qui avait une théorie selon laquelle « une femme en se mariant perd $50 \%$ de sa valeur et, quand elle a un enfant, en perd $25 \%$ de plus ». Lorsque j'ai eu la confirmation de ma seconde grossesse, il a fallu en faire part à ce supérieur qui était par ailleurs très satisfait de mon travail auprès de lui. « Eh bien, lui dis-je, je vais vous faire de la peine, parce que bientôt vous jugerez ma valeur professionnelle nulle». Devant son étonnement, je lui annonce mon état et lui rappelle sa théorie : je l'ai vu déstabilisé quelques instants et puis en rire avec moi. L'humour aide à faire passer des messages difficiles ou à sortir de situations délicates...

D'autres anecdotes donnent une idée du climat d'alors et montrent que nous plongeons les hommes dans l'embarras et qu'ils ne nous font pas confiance a priori. Clin d'œil du destin, la Marine m'amène à réaliser mon projet professionnel d'adolescente en m'affectant à l'établissement cinématographique et photographique des Armées (ECPA). Lorsque je me présente au chef d'établissement, il ne me prête aucune attention. À ses yeux, le lieutenant de vaisseau que je suis n'existe pas. C'est l'un de ses collaborateurs, en qui il a toute confiance, qui provoque son intérêt en désignant «la lumière sous le boisseau, là-bas ». A partir du moment où je suis invitée à démontrer mes compétences, je puis enfin donner toute ma mesure, et commence alors une collaboration absolument prodigieuse dans un registre qui, la passion pour le $7^{\mathrm{ème}}$ art 
aidant, décuple mes talents. Je deviens le premier officier féminin chef du département Cinéma. Je ne réalise pas les films comme j'en rêvais à quinze ans, je les produis et cela dans les meilleures conditions qui soient. Cette affectation reste très chère à mes souvenirs.

Un autre de mes « pachas » n'y va pas par quatre chemins. Au cours de notre premier entretien professionnel, il reconnait n'avoir jamais travaillé avec une femme (c'est un leitmotiv, auquel je réponds invariablement : «c'est comme avec un homme, rassurezvous, il n'y a pas de mystère »). Comme j'aurai à traiter des questions de politique de féminisation, il me déclare tout net : « Madame, la féminisation, je m'en fous (sic)». Ne voyant en moi qu'une représentante de la gent féminine, il m'ignore. Jusqu'au jour où il me fait appeler. «J'ai besoin de vous. Le contrôle général des Armées veut savoir où nous en sommes de l'expérimentation d'embarquement [des femmes], c'est vous qui allez préparer la réponse.» Comme il juge ma production de qualité, il m'accorde sa confiance et progressivement nous collaborons très efficacement.

Après être passée par l'École supérieure de guerre navale, je craignais qu'on ne reconnaisse ni mes efforts, ni le label que j'avais obtenu; je redoutais qu'on ne me confie pas un poste de brevetée. Finalement, la direction du personnel envisage pour moi un emploi très convoité par les hommes, aucun n'étant, à ce moment précis, disponible pour le tenir. On propose «LA» brevetée de l'Ecole de Guerre au chef de division qui, dans un premier temps, refuse cette éventualité. "Ah non, je n'ai rien contre elle, mais elle n'a pas le profil technico-opérationnel requis». Il finira par m'accepter dans son équipe, sans réserve cette fois, et je deviendrai pour lui une collaboratrice à part entière. C'était en 1989.

17 Cette méfiance, fondée en grande partie sur notre différence et sur la méconnaissance de nos potentialités, perdurera, m'imposant de faire mes preuves inlassablement. Il est heureux que les choses se normalisent aujourd'hui et que mes jeunes consœurs ne rencontrent plus ce genre d'obstacle.

Clio : Pourriez-vous nous parler de ces trente années au cours desquelles vous avez été associée aux changements dans la Marine concernant la place des femmes.

19 CD : Je représente la seconde génération de femmes. La première, ce sont les femmes qui se sont mobilisées après l'appel du 18 juin 1940 : en 1944, à Londres, elles sont 1100 Françaises dans la Marine; elles rendent des services exceptionnels, des services du temps de guerre, avec un statut assez sommaire. Elles ont un mérite capital à mes yeux, celui de nous avoir créé une place. Après la Libération, elles ont été renvoyées dans leurs foyers, et les armées n'en ont conservé qu'un tout petit nombre, dans des tâches traditionnellement féminines, en gros de secrétariat. Ma génération va qualifier cette place: nous n'avons pas le même profil que nos aînées, nous avons fait des études supérieures, nous voulons qu'on nous donne des choses intéressantes à faire. Nous allons conquérir des responsabilités. Et la troisième génération, celle qui «monte » à partir de 1993, n'a plus à se poser la question de la légitimité, parce que ces jeunes femmes sont recrutées et formées de la même façon que les hommes et avec eux, et qu'elles ont et auront les mêmes cursus de carrière. Ce qui ne veut pas dire pour autant que tout sera facile pour elles.

20 Je totalise trente-trois ans de services. Trente-trois ans, ce n'est rien au regard de l'Histoire, mais à l'échelle d'une vie, c'est beaucoup... Ces trois décennies sont marquées par de profonds changements, qu'on peut situer en trois étapes. 
21 Au début des années 1970, c'est le ministre Michel Debré qui donne une impulsion quantitative, ce qui forcément aura des conséquences qualitatives: les effectifs du personnel féminin triplent en six ans. En 1977, nous sommes 600 femmes, dont 23 officiers. Les choses bougent sous le coup de boutoir du politique.

La deuxième étape - le début des années 1980 - est due aussi à un ministre, Charles Hernu. Non seulement il confirme les orientations quantitatives données avant lui, mais il est l'homme de l'accélération qualitative et ouvre largement les métiers. Il ordonne à la marine une expérimentation [pendant cinq ans] d'embarquement des femmes sur les bateaux. Cette décision ministérielle, j'en témoigne, eut quasiment l'effet d'une bombe et fut pour beaucoup un véritable " traumatisme " psychologique.

Vient la dernière étape ; au début des années 1990. Là, ma contribution personnelle, une touche minuscule (permettez-moi d'être immodeste un instant), prend tout son sens. Je propose au directeur du personnel de l'époque d'ouvrir pour lui le dossier féminisation. Il me fixe rendez-vous et m'écoute pendant une heure et demie en prenant des notes. Je lui livre l'argument qui va faire mouche: "Cette nouvelle étape de féminisation, que nous pressentons comme inéluctable, ne laissons pas le politique nous l'imposer, mais prenons-en l'initiative. Il y a deux avantages à le faire : vis-à-vis du ministre, anticiper permet de combattre l'image conservatrice, voire rétrograde de la Marine. Ensuite, si le ministre accepte le plan que la Marine propose, vous pourrez le mener à votre rythme et selon les modalités que vous aurez fixées; ce qui est autrement plus confortable. » « Oui, vous avez raison ».

Il fallait pour convaincre le directeur ce jour-là que je sois crédible. Et ma crédibilité provenait du parcours que j'avais réalisé jusqu'alors, c'est-à-dire de la démonstration, affectation après affectation, de mes propres compétences, au prix d'efforts soutenus. Compétences que concrétisait emblématiquement le brevet de l'École de guerre.

L'École de guerre a été un passage fondamental, même si cette scolarité n'a pas fait de moi un marin "opérationnel ", il était bien trop tard. Ce que j'ai prouvé, avec des atouts de départ différents, c'est que je pouvais rejoindre le peloton de tête des hommes. Avec des "contre-atouts" même: femme dans un monde d'hommes, littéraire parmi des scientifiques, sédentaire quand le métier s'exerce à la mer. Par statut, je ne pouvais naviguer, et les mentalités n'étaient pas encore prêtes à accepter cette "révolution». Et voilà que j'étais admise sur concours - et bien classée - à intégrer le «Club» fermé où l'on puise les futurs dirigeants de la Marine. Ce label, j'ai été la seule à le détenir pendant treize ans, il a fallu treize années avant qu'une autre brevetée me rejoigne. Il m'a conféré une forme de légitimité qui, sans être totale, m'a offert une audience que je n'aurais pas eue sans lui. Une autre chose m'a aidée : je pense m'être fait apprécier par des prises de position modérées et sensées. Pour réussir en tant que pionnière, je l'ai dit, il ne fallait absolument pas afficher un féminisme militant. À une stratégie de la parole, j'ai préféré une stratégie de l'acte, plus discrète mais plus efficace : une brèche s'ouvre, on s'y engouffre et seulement après, on dit: «Eh bien oui, je l'ai fait.»

Clio : La politique de féminisation a-t-elle obéi au même rythme selon les armées?

$\mathrm{CD}$ : Dans l'armée de terre il y a eu de très fortes réticences, le mot est faible, à la féminisation des armes dites de mêlée. Des oppositions demeurent aujourd'hui. Quant à l'armée de l'air, jeune, moins prisonnière des traditions, elle est la plus féminisée. Elle a 
accordé rapidement une place importante aux femmes, dans des métiers qui ne sont pas accessoires. Elle les a tous, sans exception, ouverts aux femmes.

Clio : Est-ce que ça tient à la technique? L'armée de l'air comme la Marine n'ont-elles pas besoin de davantage de techniciens, d'ingénieurs à la différence de l'armée de terre où la fonction combattante occupe plus de place?

$\mathrm{CD}$ : Oui, ça joue sans doute en partie. Mais je ne pense pas que la dimension technique fasse toute la différence. La Marine est très technique, elle a beaucoup d'emplois pointus : pourtant elle s'est féminisée la toute dernière. Il faut chercher la réponse du côté du milieu où le métier s'exerce. C'est un argument que j'ai parfois opposé à des interlocutrices de valeur, comme le médecin général Valérie André quand elle présidait la commission d'études prospectives de la femme militaire, ou Edwige Avice, secrétaire d'État à la Défense. "Vous ne pouvez pas gommer les spécificités du milieu où nous agissons. Il faut naviguer pour comprendre la Marine et les marins. Même si les bateaux sont plus confortables qu'autrefois, les conditions de vie à bord peuvent expliquer que les femmes aient été si longtemps tenues à l'écart». Il y a des raisons objectives : l'inconfort, la fatigue, la promiscuité. Ce sont des métiers qui usent prématurément parce que vous travaillez sur une plate-forme mouvante, c'est-à-dire qu'une partie de votre énergie est brûlée par la compensation permanente des mouvements de plateforme, et le reste par votre activité professionnelle. Il y a aussi l'éloignement et la durée des missions, souvent imprévisible. Il y a enfin la nécessité de maintenir la cohésion de l'équipage dans la durée. Bref, un faisceau parfaitement objectif peut justifier que la Marine ait réfléchi à deux fois avant d'ouvrir ses bateaux à des équipages mixtes.

Clio : Comment la Marine a-t-elle anticipé l'arrivée des femmes sur les bâtiments?

$\mathrm{CD}:$ Ah, cette préparation fut vraiment passionnante. Comment avons-nous procédé ? Après mon entretien avec le directeur du personnel, en 1991, le haut commandement de la Marine a créé un groupe de travail mixte, composé de huit officiers, quatre hommes et quatre femmes, choisis en fonction de la variété de leur expérience. Parmi les quatre femmes, j'étais la plus ancienne. Les trois autres étaient: un capitaine de corvette qui avait une courte expérience à la mer comme officier de renseignement ; un jeune lieutenant de vaisseau qui avait navigué dans le cadre de l'expérimentation et serait ensuite l'une des premières à commander à la mer; et un jeune enseigne avec un profil d'officier marinier devenu officier par le rang. Et les hommes? C'était un panel de capitaines de vaisseau et de frégate, plus homogène. Pendant un an, nous avons travaillé dur sur ce dossier, en plus de nos fonctions respectives. Ce qui a été troublant et amusant à la fois, c'est que, sans nous concerter, nous avons toutes les quatre abordé ce travail avec la même volonté : "Il faut que ça bouge, nous avons la chance de faire partie d'une instance de travail qui va en principe améliorer notre condition, il faut donc des résultats. " Face à nous, les quatre messieurs semblaient un peu sceptiques par rapport au thème de l'étude. Au fur à mesure que nous avancions, nos confrères étaient de plus en plus convaincus et " progressistes». Nous, les femmes, étions moins assurées et avions tendance à les freiner : «Attendez, croyez-vous que l'on puisse aller aussi loin?»

Nous avons pris le temps de bâtir un dossier solide, de ne rien laisser au hasard. Nous sommes allés dans l'éducation nationale voir comment les jeunes filles s'orientaient vers les métiers de la technique, vérifier que le vivier existait bien, faute de quoi il est vain d'ouvrir toutes les voies puisqu'il n'y aura pas de ressource pour les alimenter. On a regardé à quel âge les jeunes Françaises avaient leur premier enfant : vers 28 ans et 
demi - 29 ans. Le calcul était simple à faire : 28 - 18, soit dix ans pendant lesquels elles ne seraient pas entravées par des attaches familiales. Elles assuraient ainsi à la Marine assez de disponibilité pour recevoir une ou deux affectations à la mer. On a aussi pris en considération les évolutions de la démographie. On a consulté également trois marines étrangères - celles des États-Unis, des Pays-Bas et de Grande-Bretagne - pour nous inspirer des bonnes choses qu'elles avaient faites et éviter les écueils dans lesquels elles s'étaient fourvoyées. Par exemple, le pourcentage optimal de femmes à bord. Les Américains et les Hollandais avaient commis l'erreur de féminiser d'emblée à $50 \%$ certains de leurs bateaux. C'est une aberration! Cela s'est très mal passé. Les Américains ont réétudié sérieusement la question et ont déterminé la fourchette optimale - entre 10 et $25 \%$ - pour une intégration harmonieuse des femmes. C'est le pourcentage plancher (10\%) que nous avons retenu pour notre étude. Il confirmait ce que nous avions constaté par nous-mêmes durant l'expérimentation d'embarquement. Menée entre janvier 83 et décembre 87, cette expérience avait enregistré le volontariat de quarante femmes seulement sur un total de 1700 . Ce qui valait pour 40 ne pouvait être étendu à la totalité. Le rapport au ministre avait conclu : «Si qualitativement, cela s'est très bien passé, quantitativement, le résultat n'est pas significatif. » Certes, il y a eu quelques problèmes relationnels qui ont trouvé leur solution. L'expérimentation a surtout prouvé que les deux communautés en présence à bord étaient capables de faire l'effort de s'accepter et de se respecter mutuellement. On était fin 1987, les esprits n'étaient pas encore mûrs. Les décideurs estimaient que les efforts consentis avaient été bien trop lourds pour un résultat quantitatif si mineur : "Pourquoi nous imposer des éléments perturbateurs à bord, quand nos bateaux marchent très bien sans eux? " Ils n'avaient pas tort, eu égard au premier devoir d'un commandant qui est de maintenir la cohésion de son équipage envers et contre tout. Toutefois, ce raisonnement avait le défaut de ne pas aller dans le sens de l'Histoire...

Clio: Quelles mesures d'organisation ont été prises en préparation de l'embarquement de femmes?

CD : Pendant la période expérimentale, on a embarqué les femmes sur les bateaux tels qu'ils étaient. C'est-à-dire sans les modifier à cette fin. Comme je l'ai dit, elles sont venues en tout petit nombre et ont été affectées sur les bâtiments selon leur spécialisation. Elles étaient une, deux ou trois à bord. Si ne rien aménager à leur profit permettait de conduire l'expérience dans des conditions réalistes, j'imagine ce que cela a dû être psychologiquement, notamment pour celles qui étaient seules sur leur navire. capacités y a eu de gros bateaux (porte-avions, TCD), parce qu'ils avaient de bonnes capacités de logement, mais également des petits bâtiments (chasseurs de mines). Cela
a conduit parfois à faire loger un homme et une femme dans la même chambre. Outre la difficulté à gérer cette proximité, s'est posé le problème de l'attitude des épouses. Le lobby des épouses avait été très puissant outre-Manche. En France, il a développé les mêmes arguments : "Pourquoi tenter nos maris pendant les temps de navigation? les escales n'étaient-elles pas une épreuve suffisante ?...»Ces résistances me semblent naturelles et compréhensibles. Il a fallu expliquer et communiquer.

Clio : Il n'a pas été décidé un aménagement spécifique des navires?

CD : C'est un des grands enseignements tirés de l'expérimentation et, plus tard, de nos missions dans les marines étrangères. En particulier, on l'avait observé sur les navires

Clio. Femmes, Genre, Histoire, 20 | 2004 
américains qui réservaient aux femmes des quartiers où elles disposaient de leurs chambres et des locaux d'hygiène associés, le tout préservant leur intimité. À partir de nos recommandations, il est décidé en 1992 - l'année 1993 étant la première année d'application de la mixité - de ne féminiser un bâtiment que lorsqu'il a été aménagé (ou conçu dès l'origine) pour accueillir du personnel féminin. La mixité des équipages ne se réalise donc qu'au rythme des IPER des bâtiments, c'est-à-dire des périodes d'entretien et de réparation qui les immobilisent plusieurs fois dans leur vie. Cette lenteur, que certains ont voulu interpréter comme un manque d'empressement, est la condition-clé du succès : une fois un bateau décrété mixte, c'est acquis définitivement. Nous avons évité les désastreux retours en arrière des États-Unis, de la Grande-Bretagne ou des Pays-Bas.

Clio: Vous préparez la mixité selon des quotas ? C'est-à-dire le navire peut-il accueillir d'emblée moitié de femmes ou seulement selon une fourchette rigide?

$\mathrm{CD}$ : Pour ne pas commettre les erreurs de ceux qui nous ont précédés, on a adopté au départ la valeur plancher de $10 \%$; les choses se passant bien et le nombre des femmes ayant augmenté, nous la portons aujourd'hui à $15 \%$. Les bateaux qui ont subi des aménagements au cours de leurs refontes et réparations peuvent «absorber" ce supplément car on s'était octroyé une petite marge de manoeuvre.

Clio: Ce qui sous-entend qu'il faudra les réaménager si la part des femmes progresse?

CD : Il le faudrait si on voulait affecter davantage de femmes à bord. Mais faudra-t-il aller jusqu'au pourcentage plafond $(25 \%)$ ? On peut aussi augmenter le nombre des bâtiments à équipage mixte ${ }^{1}$. Je sais qu'il y a une forte demande de la part des très jeunes femmes. Elles veulent toutes naviguer en début de carrière car elles se sentent disponibles pour le faire et l'embarquement constitue en soi un facteur d'attractivité du métier non négligeable. Maintenant que tous les emplois sont ouverts, sauf ceux qui s'exercent à bord des sous-marins mais j'y reviendrai, ne pas pouvoir naviguer dans certains métiers, nautiques en particulier, constituerait un préjudice de carrière. Il faut faire très attention à ne pas pénaliser les femmes dans l'acquisition de leurs compétences ni dans leur avancement. Hommes et femmes ont droit à l'équité.

Quand on a rendu mixtes les équipages, on a aussi imposé des règles de vie et de comportement à bord. Quand je relis ces écrits, qui mettent les points sur les i, ils me paraissent un peu surréalistes.

Dès 1993, les équipages de deux frégates ont été féminisés. Les deux commandants ont eu droit à des briefings et à des recommandations en bonne et due forme. L'un semblait assez serein, l'autre plus inquiet mais je suis certaine qu'ils n'en menaient pas large ni l'un ni l'autre. Il y avait une appréhension forte, tout à fait compréhensible : comment les choses allaient-elles se passer? Les choses se sont bien passées : on a alors senti une espèce de soulagement extraordinaire de toute l'institution: " ça fonctionne, ça se passe bien, il n'y a pas de problème ! » Les craintes apaisées ou dissipées, une autre idée s'est fait jour, celle de la banalisation. La mixité des équipages était devenue banale. Personnellement, je trouve le mot malheureux et je m'inscris en faux contre l'idée qu'il recouvre: les relations masculin-féminin, dans la Marine comme ailleurs, ne seront jamais banales. Elles tendent à devenir naturelles (les précautions réglementaires de 1993 me semblent aujourd'hui dépassées, ce qui indique une évolution normale des mentalités). J'ai beaucoup incité à une vigilance de bon aloi, car il peut toujours se produire quelque chose de fâcheux, surtout dans le champ clos du bateau. 

réussite, c'est d'avoir à bord une représentation de toute la hiérarchie des grades. En Grande-Bretagne, notre groupe d'étude avait pu naviguer une journée sur la frégate Southampton. Il y avait à bord 250 personnes, dont vingt femmes : deux officiers et dixhuit matelots. Nous avons eu le droit de circuler à bord très librement. Quand nous interrogions, dans leurs carrés respectifs, les officiers mariniers masculins britanniques, ils nous répondaient: « Les femmes à bord? On ne les voit pas et on ne veut pas les voir. Elles sont matelots et n'ont pas accès à nos carrés, à nos lieux de détente, qu'elles vivent leur vie!» Avec les officiers subalternes, c'était un peu différent parce qu'ils accueillaient les deux officiers féminins. Nous en avons déduit que si nous voulions que les femmes soient intégrées, il fallait que leur présence se diffuse non seulement dans les équipes de travail, mais aussi dans les carrés. Vous savez, le bateau est un lieu où vous travaillez et où vous vivez, contrairement à la terre où le lieu de travail est différent du lieu de vie. Nous avons donc inscrit une condition supplémentaire dans nos recommandations: la représentation de toute la hiérarchie des grades pour le personnel non officier. Cette condition a bien sûr freiné le mouvement. En effet, nous disposions, en 1993 et dans les années qui ont suivi, d'un nombre important de jeunes femmes dans les premiers grades mais de très peu d'officiers mariniers supérieurs, volontaires pour naviguer. Nous devions effectivement recourir aux anciennes qui ne s'étaient pas engagées dans cette perspective. Et aujourd'hui, dix ans plus tard, nous avons encore du mal à trouver cette maistrance supérieure féminine. Progressivement, la situation trouve son régime d'équilibre.

Pour les raisons que je vous ai dites, notamment le rythme de féminisation des équipages induit par les modifications des bords et le préjudice de carrière qui aurait pu affecter le personnel féminin, on a décidé de rendre mixte le porte-avions Charles de Gaulle un peu plus vite que prévu.

Clio : De ce fait, la moitié des femmes embarquées sont sur le Charles de Gaulle?

CD : Oui, cette décision, conforme au pourcentage fixé, permettait d'embarquer d'un seul coup un grand groupe de femmes, bénéficiant de la présence d'officiers féminins en nombre significatif là aussi. Dans la réflexion qui est menée depuis six mois, la Marine envisage d'assouplir encore les règlements pour permettre à plus de femmes de naviguer : accès à davantage de bateaux, y compris de plus faible tonnage, pourcentage un peu plus important de jeunes femmes que d'anciennes sur les bateaux qui sont mixtes depuis un certain temps déjà, c'est-à-dire dont l'équipage est désormais familiarisé avec cette dimension-là. On étudie par ailleurs la possibilité d'embarquer les couples mariés sur le même navire.

Clio : L'armée c'est aussi le combat, est-ce que l'entraînement est mixte?

Clio: L'institution militaire a-t-elle aujourd'hui une doctrine sur la place des femmes au combat? 

femmes ne servent pas en première ligne. Pas la Marine. Sur un bateau, qui constitue une entité, il serait extrêmement difficile de faire des distinguos : si la plate-forme est en avarie de combat, tout l'équipage est concerné de la même façon. Nous opérons dans un milieu hostile, la mer. La sécurité est l'affaire de tous. Chacun, homme ou femme, sait parfaitement ce qu'il doit faire en temps de combat. Les entraînements servent à créer ces automatismes. Si le bâtiment est touché, s'il sombre, le risque est partagé par tous.

de vue lors du SIGEM, le séminaire interarmées destiné à 600 élèves officiers des grandes écoles militaires pendant quinze jours à Paris. Il estimait que la présence des femmes au combat ne serait jamais neutre et pèserait sur l'équipe où elles se trouveraient. Considérées comme les maillons faibles, elles détourneraient les hommes de l'accomplissement de leur mission puisqu'en cas de blessure, comme dans le risque d'une capture, ces derniers seraient naturellement occupés à leur porter assistance. On m'a rapporté cette intervention qui a suscité des remous dans l'assistance et des protestations de la part des élèves féminins.

60 Mais si j'avais pu discuter avec cet officier, je lui aurais dit que ses affirmations ne sont pas démontrées, qu'il faut compter avec le jusqu'au-boutisme des femmes, avec leur agressivité et leur violence, avec leur cran et leur résistance morale - pour la force physique, il est vrai que le rapport reste bien en faveur du masculin, c'est une donnée de la nature. Je ne suis absolument pas sûre que, à terre dans les conditions extrêmes du combat, ce ne soit pas le chacun pour soi qui l'emporte.

61 Les jeunes filles qui s'engagent aujourd'hui dans ce métier, encore très masculin, le choisissent parce qu'elles ont envie de s'exercer sur un terrain réputé moins facile que celui des professions "asexuées ». Elles sont capables d'une grande énergie, voire de violence. Il est temps de ne plus les considérer comme des bibelots fragiles, dans les rôles où le XIX ${ }^{\mathrm{e}}$ siècle s'est plu à enfermer les femmes.

62 Il subsiste pourtant des conduites, des attitudes masculines que je trouve désuètes. Il existe encore des rites de passage dont le prix est trop élevé. Par exemple, une jeune mécanicienne travaillant dans un atelier devra peut-être gommer sa féminité pour se faire admettre de son entourage masculin et faire reconnaître sa compétence technique, c'est dommage.

Mais, l'avantage considérable par rapport à ce que j'ai connu il y a trente ans, c'est notre nombre ${ }^{3}$ : un groupe de bonne taille, même minoritaire, est visible, il existe. Il a de l'influence et rééquilibre le rapport de force en relativisant la logique dominées/ dominants. J'aime à penser, comme ce fut mon cas, que la plupart des femmes 
comprennent qu'à chaque fois qu'elle font quelque chose, elles engagent la crédibilité du groupe auxquelles elles appartiennent.

Clio: La suspension du service national a-t-elle eu un impact sur l'évolution du recrutement de femmes?

CD : L'annonce en est faite en 1996 et la réalisation de 1997 à 2002. Fin 2002, il n'y a plus d'appelés dans les armées. Or, il est trop tôt pour mesurer l'impact de cette décision importante. Pour nous, la professionnalisation se fait davantage sentir maintenant que durant la période de décroissance et de disparition progressive des appelés. Il n'y a pas eu de rupture brutale.

L'arrivée en grand nombre des civils - aujourd'hui un marin sur cinq est civil, c'est important car il n'y en a pas à bord des bateaux, le taux à terre peut atteindre $40 \%$ représente un changement culturel, une autre façon de diriger. Le recrutement de jeunes sans qualification pour remplacer certains appelés, a également modifié le paysage humain. On peut se demander comment évoluera notre recrutement et quelle y sera la place des femmes. Si elle s'accroît, compte tenu de la grande qualité des candidatures féminines, ce sera un contrat gagnant-gagnant, pour l'institution d'une part et pour la nation de l'autre. Si les candidats se détournaient de la Marine, on serait assez naturellement tenté de se tourner vers elles, sans pâtir des limitations d'autrefois, quand les femmes ne naviguaient pas. Je pense que le nombre de femmes est appelé à augmenter. Mais pas au-delà d'un maximum que je situe vers $20 \%$. Pourquoi? Parce qu'il existe des régulations naturelles: par exemple, réussir une carrière longue induit de passer par l'École Navale, c'est-à-dire par les classes préparatoires aux grandes écoles. Or, dans les formations scientifiques, les filles ne sont pas plus de $17 \%$. Autre régulation, la contrainte du métier, la notion de disponibilité dans la durée. Actuellement nos statuts sont revus. La Marine a fait des propositions pour les assouplir un peu, c'est-à-dire aménager le temps de travail: c'est une condition si nous ne voulons pas nous priver de l'apport des femmes. Tout cela est en débat, rien n'est décidé. Mais, sans aller jusqu'au temps partiel qui fragiliserait l'une de nos spécificités militaires, la disponibilité, je crois qu'il faudra trouver, inventer des formules qui permettent aux femmes de mieux concilier le temps de la vie personnelle et le temps de la vie professionnelle. Sinon, nous ne pourrons pas les fidéliser.

\section{Clio: Cela vaut aussi pour les hommes?}

$\mathrm{CD}$ : J'allais le dire. Tout ce qui sera proposé dorénavant dans le domaine des droits et des devoirs s'appliquera aux deux sexes sans distinction, loi oblige. Les facilités qu'on accordera, iront aussi bien aux hommes qu'aux femmes. Le moyen de résoudre la contradiction temps de travail/disponibilité $\mathrm{H} 24$ proposé par la Marine consiste en un congé d'éducation, à l'instar de ce qui existe dans la société civile, à la différence qu'il ne serait pas acquis de droit, mais accordé par la hiérarchie en fonction de l'activité opérationnelle et resterait révocable à tout moment si la situation l'exige. Il convient de tenter des modes d'action nouveaux, d'inventer des règlements qui correspondent à des situations nouvelles. Pour moi, agir ainsi s'apparente plus à une évolution naturelle de l'institution militaire sous l'impact des changements sociétaux qu'à une révolution de l'état de militaire. Il serait dommage de connaître ce que l'on a vu aux États-Unis ou aux Pays-Bas, c'est-à-dire des femmes qui font un passage court dans la marine faute d'arriver à trouver un équilibre de vie. Je vous disais au début de l'entretien que j'ai eu la chance et la malchance de ne pas naviguer. Chance car cela m'a évité la contrainte de devoir répondre présente à tous les rendez-vous embarqués que l'institution m'aurait 
fixés dans la durée. Malchance parce que le cœur du métier s'exerce à la mer. J'ai eu accès à l'embarquement trop tard pour faire une carrière normale et bien tard pour décoder et comprendre des comportements, des attitudes, des traditions auxquels seule la navigation donne tout leur sens. Il faut le vivre pour le comprendre.

La question de l'embarquement ne se posant pas, j'ai eu à gérer la carrière de toute femme qui accède aux responsabilités et qui doit s'organiser pour élever ses enfants sans trop d'à-coups. Comme d'autres, j'ai connu l'écartèlement de devoir confier un bébé à des mains étrangères, le bénéfice d'un congé sans solde ne nous étant pas encore autorisé à l'époque. Comme d'autres, je me suis gendarmée pour consacrer à mes enfants le maximum de mon temps après la journée de travail, quelle que soit ma fatigue. Les jeunes femmes demain auront à caser l'embarquement dans leur équation personnelle et ce sera sans doute une lourde responsabilité.

Clio : Une question un peu personnelle : vous avez eu un parcours de pionnière, vous avez ouvert des portes, permis que des portes s'ouvrent aussi, et de ces trente années passées vous retirez certainement une fierté d'avoir fait bouger les choses. Peut-être avez-vous aussi des regrets sur certains points, des opportunités vous ont été refusées parce qu'à l'époque elles n'étaient pas autorisées aux femmes, mais, par votre parcours, vous allez permettre à d'autres de les vivre?

71 CD : J'ai ressenti comme un honneur d'être la première à incarner l'amiralat. Une grande fierté aussi. En fait, je ne sais pas bien juger de mon parcours, c'est ce que les autres m'en montrent, les miroirs qu'ils me tendent - par exemple l'admiration que je lis dans les yeux des jeunettes ou les succès d'estime que je remporte auprès des publics variés auxquels je m'adresse - c'est tout cela qui me donne à penser que mon parcours a été plus remarquable que je ne l'ai éprouvé jour après jour. Les années, les situations de ma vie d'officier, d'amiral même, n'ont pas été, ne sont pas forcément des situations d'exception, il y a beaucoup d'événements banals ou pesants dans le quotidien. Certes, il y a des circonstances insolites ou plus exaltantes. Mais, je n'ai pas vécu dans un état de grâce permanent! Le plus lourd à supporter reste le regard des autres, c'est de travailler sous stress constant. Quand je quitterai mon tablier d'amiral, je suis sûre de gagner sur ce point.

Des regrets ? D'abord je n'en ai pas par principe, c'est trop stérile. Ce que je peux dire, c'est que si dans le journal de ma région natale, le Val de Loire, j'avais lu « recrutement d'officiers féminins navigants ", je ne me serais pas portée candidate. J'étais trop concentrée sur mon projet professionnel, faire du cinéma, pour aller travailler sur la mer. J'ai lu « recrutement d'officiers de marine ». J'ai simplement demandé à voir et j'ai choisi cette option parce qu'elle me permettait d'aller vers le cinéma. Et une fois dans la Marine, j'ai eu le sentiment que mon sort était étroitement mêlé au destin du groupe de femmes auquel j'appartenais. Cette circonstance particulière a donné à mon parcours une assise peu ordinaire. En même temps, elle a pesé d'un poids, lui aussi, extraordinaire. La Marine m'a demandé d'être exemplaire, elle aura été pour moi une école de rigueur et d'exigence jusqu'au terme de mon cursus. Si j'y suis parvenue, c'est sans doute parce que j'avais pas mal de répondant en moi. L'important est de se réaliser, n'est-ce pas?

Je dis souvent que ma vie professionnelle illustre ce mot de Valéry que j'aime beaucoup : «Fais de tes faiblesses une force qui te servira." Se construire, s'affirmer dans l'adversité, prendre l'avantage sur soi-même et pour soi-même. Ne pas se résigner, rebondir en cas d'échec, et toujours avancer, parce que la vie est mouvement, et que l'immobilité est la mort. Accueillir toutes les affectations comme des défis, faire un 
formidable métier où l'on touche à des domaines si différents et où l'on peut apporter des réponses si différentes. C'est ce que j'ai essayé de faire, modestement.

\section{NOTES}

1. Onze bâtiments sont mixtes aujourd'hui.

2. La prochaine génération de sous-marins nucléaires d'attaque, du type Barracuda, a été conçue pour recevoir des équipages mixtes. Elle devrait être mise en service dans une dizaine d'années.

3. En 2004, la Marine compte 5000 femmes dont 470 officiers, soit un taux global de $11,5 \%$.

\section{RÉSUMÉS}

Entrée comme officier dans la Marine en 1970, affectée en 1983 à la Direction du personnel, Chantal Desbordes participe à la politique de féminisation des effectifs. Première femme brevetée de l'Ecole supérieure de Guerre navale elle est nommée contre-amiral en conseil des ministres du 19 décembre 2001. Elle est ainsi le premier officier général féminin dans la Marine française. Actuellement adjointe au directeur du personnel militaire de la Marine, elle est chargée de la gestion des compétences des marins. L'entretien de deux heures, réalisé le 24 octobre 2003 au ministère de la Défense (Paris), portait sur sa trajectoire personnelle et sur le développement du recrutement féminin au sein de la Marine. Nous en publions ici quelques extraits. 